\title{
VEGETACIÓN Y FLORA DE UN ECOTONO ENTRE LAS PROVINCIAS DEL ALTIPLANO Y DE LA PLANICIE COSTERA DEL NORESTE DE MÉXICO
}

\author{
OSCAR BRIONES \\ Instituto de Ecología, A.C. \\ Apartado postal 63 \\ 91070 Xalapa, Veracruz \\ Correo electrónico: briones@ecologia.edu.mx \\ Y \\ J. A. Villarreal Q. \\ Departamento de Botánica \\ Universidad Autónoma Agraria Antonio Narro \\ Buenavista, \\ 25315 Saltillo, Coahuila \\ Correo electrónico: javillarreal@correoweb.com
}

\section{RESUMEN}

Se estudió la vegetación y la flora vascular de un ecotono entre las provincias florísticas del Altiplano y de la Planicie Costera del Noreste de México. El área de estudio cubre una superficie de $2,526.25 \mathrm{~km}^{2}$ en el norte del estado de Nuevo León.

Con base en 50 muestreos de vegetación, fotografías aéreas y colectas botánicas, se delimitaron y cartografiaron siete tipos de vegetación, mismos que se describen de acuerdo con su fisonomía, características ecológicas y componentes florísticos. Se determinaron 405 especies, incluyendo variedades y subespecies. La zona presentó afinidad geográfica con la vegetación del Desierto Chihuahuense en el Altiplano (pastizal de Hilaria mutica, matorral bajo inerme de Flourensia cernua y matorral mediano de Acacia-Prosopis-Flourensia-Larrea) y la Planicie Costera (matorral mediano subinerme de Leucophyllum frutescens y Acacia rigidula y matorral alto subinerme de Helietta parvifolia). Los matorrales de Prosopis-Acacia no mostraron afinidad geográfica definida.

La coexistencia de vegetación arbustiva característica de las tierras bajas de la Planicie Costera, con la vegetación comúnmente encontrada en el Desierto Chihuahuense, podría explicarse en virtud del incremento en la aridez y el suave levantamiento de la superficie continental en sentido este-oeste y de la ausencia de accidentes topográficos notables en el sector nororiental de México.

Palabras clave: zonas áridas, Desierto Chihuahuense, matorral espinoso tamaulipeco, fitogeografía, noreste de México. 


\section{ABSTRACT}

The vegetation and the vascular plants of an ecotonal zone between the floristic provinces of the Desert Plateau (Chihuahuan Desert) and Gulf Coastal Plain in northeastern México were studied. The study zone has a surface of $2,526.25 \mathrm{~km}^{2}$ and it is located in the northern part of the state of Nuevo León.

On the basis of physiognomy, ecological characteristics and dominant species, seven types of vegetation were recognized. With the aid of 50 sampling plots, aerial photographs and floristic composition, the units of vegetation were delimited and mapped. 405 species (varieties and subspecies included) were identified. The study zone showed geographical affinity with the vegetation of the Chihuahuan Desert in the Desert Plateau of México (Hilaria mutica grassland, low thornless scrub of Flourensia cernua and medium size mixed scrub of Acacia-Prosopis-Flourensia-Larrea) and with the Gulf Coastal Plain (medium size mixed scrub of Leucophyllum frutescens and Acacia rigidula and high mixed scrub of Helietta parvifolia). The medium size and high thorny scrubs of Prosopis-Acacia showed no clear relationships with any of the floristic provinces.

The coexistence of shrubby vegetation characteristic of the lowlands in the Gulf Coastal Plain and the vegetation usually present in the Chihuahuan Desert, can be explained in view of the increment of aridity and gentle raising of the continental surface in east-west direction, and of the absence of notable topographic accidents in the northeastern region of México.

Key words: arid zones, Chihuahuan Desert, Tamaulipan thorn scrub, phytogeography, northeastern Mexico.

\section{INTRODUCCIÓN}

Los autores que han aportado información sobre la vegetación del área de estudio mencionan que en ésta se desarrollan comunidades vegetales caraterísticas de la Planicie Costera del Noreste de México (Rzedowski, 1978). Muller (1939) definió para el norte del estado de Nuevo León el matorral espinoso tamaulipeco, caracterizado en el extremo septentrional de la entidad por Prosopis chilense y Opuntia sp. formando una sabana con Bouteloua trifida, Aristida purpurea, Triodia pilosa y T. texana. Edwards (1939) señaló que hacia la parte norte de la Sierra Madre Oriental se presenta una asociación desértica arbustiva de Acacia-Prosopis en la planicie y una vegetación arbustiva dominada por Helietta parvifolia en las colinas bajas. Rojas-Mendoza (1965), en su trabajo sobre la vegetación de Nuevo León, indicó que en la zona se presentan matorrales bajos subperennifolios con Acacia-Leucophyllum-Cordia, matorrales micrófilo-suculentos con Prosopis-Bouteloua-Opuntia, matorrales bajos subcaducifolios con Cordia-Acacia-Karwinskia y matorrales altos subperennifolios con Acacia-Pithecellobium-Helietta. La zona ha sido ubicada como parte de la provincia florística de la Planicie Costera del Noreste, en la región florística Xerofítica Mexicana del reino florístico Neotropical (Rzedowski, 1978).

La presencia de asociaciones vegetales características del Altiplano en la zona no ha sido señalada, con excepción de la monografía realizada por la Comisión Técnico Consultiva para la Determinación Regional de los Coeficientes de Agostadero en el estado de Nuevo León (Anónimo, 1973), en la que se describen matorrales de Larrea tridentata y pastizales de Hilaria mutica. Estas dos comunidades caracterizan a la vegetación de amplias zonas del Desierto Chihuahuense (Rzedowski, 1978). Además, algunos autores indirectamente han indicado la posible presencia de comunidades propias del Desierto 
Chihuahuense, al mencionar que en el noreste de México existe una zona de transición muy gradual entre la vegetación del Altiplano y la Planicie Costera del Noreste de México (Miranda y Hernández X., 1963b; Rojas-Mendoza, 1965; Rzedowski, 1978). Sin embargo, la incompleta información sobre la flora de la región y las comunidades vegetales que conforman, ha ocasionado que el ecotono entre ambas regiones haya sido difícil de precisar (Rzedowski, 1978).

Los objetivos de este trabajo fueron los de reconocer fisonómicamente los tipos de vegetación y representarlos en un mapa, así como caracterizar sus afinidades geográficas y elementos florísticos, en una región ubicada sobre el límite entre las provincias florísticas del Altiplano y de la Planicie Costera del Noreste de México (Rzedowski, 1978). Dichas unidades florísticas corresponden, en ese orden, a las regiones fisiográficas denominadas Gulf Coastal Plain y Anahuac Desert Plateau (Thayer, 1916) y también a las provincias bióticas definidas como Tamaulipan y Chihuahuan (Dice, 1943).

Una versión preliminar de este trabajo, carente de mapa y de lista florística, fue publicada por el primer autor bajo el título: "Notas geográficas sobre la vegetación y flora de Lampazos de Naranjo, Nuevo León, México", en Reporte Científico Número 4 (1986). Facultad de Silvicultura y Manejo de Recursos Naturales. Universidad Autónoma de Nuevo León: 20 pp.

\section{MATERIALES Y MÉTODOS}

Área de estudio

El área de estudio corresponde a la mayor parte de la superficie del municipio de Lampazos de Naranjo, Nuevo León, de la que se excluyen las partes montañosas. De acuerdo con la Carta Regionalización Fisiográfica de la Síntesis Geográfica de Nuevo León (Anónimo, 1981), se localiza en la provincia fisiográfica de la Gran Llanura de Norteamérica. La altitud varía de 200 a 500 m en las partes planas y de 350 a $850 \mathrm{~m}$ en los lomeríos y mesetas. Su situación geográfica está dada por las coordenadas $26^{\circ} 46^{\prime}$ y $27^{\circ} 24^{\prime}$ de latitud norte y $100^{\circ} 04^{\prime}$ y $100^{\circ} 50^{\prime}$ de longitud oeste. Ocupa una superficie aproximada de $2,526.25 \mathrm{~km}^{2}$.

En la estación meteorológica de Lampazos de Naranjo (Comisión Nacional del Agua, datos no publicados), situada a $302 \mathrm{~m}$ de altitud, la temperatura media anual es de $22.2^{\circ} \mathrm{C}$, en 22 años de registro. La temperatura máxima promedio de junio es de $41.0^{\circ} \mathrm{C}$ y la mínima promedio de enero es de $-1.2^{\circ} \mathrm{C}$. Temperaturas por debajo de $0^{\circ} \mathrm{C}$ se presentan entre octubre y marzo. La precipitación total anual es de $398.7 \mathrm{~mm}$ (25 años), siendo septiembre el mes más lluvioso $(92.4 \mathrm{~mm})$ y febrero el más seco $(7.7 \mathrm{~mm})$. Las principales lluvias caen entre mayo y septiembre, y entre junio y julio se presenta una corta sequía intraestival. La evaporación total anual es de 2,660.6 mm (6 años de registro), con la máxima en mayo $(480.9 \mathrm{~mm})$ y la mínima en enero $(56.4 \mathrm{~mm})$. Los vientos dominantes provienen del noreste y durante el invierno también se presentan vientos fríos procedentes del norte.

El subsuelo, de un mar de poca profundidad en el este de Nuevo León que incluye a la zona de estudio, se convirtió en tierra firme a fines del Mioceno o principios del Plioceno (Mullerried, 1944). Las rocas más antiguas que afloran pertenecen al Cretácico Superior 
y son asociaciones de lutitas y areniscas y de calizas y lutitas. Hay afloramientos de sedimentos marinos del Terciario (Plioceno), constituidos por conglomerados. Del Cuaternario se encuentran los depósitos aluviales (Mullerried, 1944; Anónimo, 1981). Los suelos son marcadamente de textura arcillosa, ligeramente alcalinos y presentan con frecuencia una capa cálcica en el subsuelo; son de color café o gris, variando de claros a muy oscuros y la materia orgánica es baja a media en cantidad. Se presentan los suelos de tipo xerosol, litosol, vertisol, rendzina y fluvisol (Anónimo, 1981). El río Candela es la única corriente permanente en la zona de estudio, corre de sur a noreste y se une al río Salado en el límite de la zona con el municipio de Anáhuac.

Métodos

Con base en fotos aéreas monocromáticas a escala 1:50,000 o 1:70,000 se marcaron 50 sitios de muestreo. En cada sitio se tomaron los siguientes datos: relieve, exposición y pendiente y en una parcela de $100 \mathrm{~m}^{2}$ se estimó visualmente el porcentaje de cobertura vegetal, la altura de los estratos de la vegetación y el índice de abundancia-cobertura para las especies de acuerdo con la escala de Braun-Blanquet (1979). Dicha escala tiene los siguientes valores: +, presente en forma dispersa, cobertura muy baja; 1 , abundante, pero cobertura baja; 2 , muy numerosa, o cobertura menor de 1/20 de la superficie; 3 , cobertura de $1 / 4$ a $1 / 2$ de la superficie; 4, cobertura de $1 / 2$ a $3 / 4$ de la superficie; 5 , cobertura mayor de $3 / 4$ de la superficie. Para distinguir y nombrar a los tipos de vegetación se recurrió a la fisonomía de la vegetación y especies dominantes del estrato superior, basándose fundamentalmente en la nomenclatura de Rzedowski (1961) y Miranda y Hernández X. (1963a). Para conocer la flora riparia y arvense, se realizaron colectas en la orilla de las corrientes superficiales y de los cultivos.

Los tipos de vegetación se delimitaron sobre las fotos aéreas y se trazaron sobre cartas topográficas a escala 1:50,000, correspondientes al área de estudio (Anónimo, 1974, 1976 y 1977). Las cartas tienen proyección UTM de la zona 14 y datum NAD27. El trabajo de campo se realizó entre febrero de 1980 y marzo de 1983. Para obtener el mapa de vegetación se siguió la metodología sugerida por Kuchler (1967). Los primeros recorridos de campo y los antecedentes florísticos del lugar permitieron trazar límites preliminares de los tipos de vegetación sobre las fotos aéreas y ubicar los sitios de muestreo. Los subsecuentes recorridos de campo, las colectas botánicas y los datos de los muestreos ayudaron a verificar y corregir la delimitación de las unidades de vegetación. Para obtener el mapa anexo, las líneas que sobre las fotos aéreas delimitaron a los tipos de vegetación, fueron manualmente trasladadas a las cartas topográficas. Los arroyos, caminos, represas, habitaciones y otras marcas sobre el terreno ayudaron a realizar la transferencia de la información. Este procedimiento permitió utilizar fotos aéreas de diferentes escalas. Finalmente, la información de las cartas topográficas fue digitizada, etiquetada y editada en ArcView 3.1. No se validó en el campo la información cartográfica una vez terminado el mapa de vegetación. Se consideró suficiente la continua corrección de la correspondencia entre la información de las fotos aéreas, las cartas topográficas y los muestreos de la vegetación realizados durante el trabajo. 
Briones y Villarreal: Vegetación y Flora de un Ecotono entre dos Provincias Florísticas de México

\section{RESULTADOS Y DISCUSIÓN}

Tipos de vegetación

Los siete tipos de vegetación reconocidos para la zona ocupan la siguiente superficie relativa, de un total aproximado de $2,526.25 \mathrm{~km}^{2}$ :

Tipo de Vegetación

Superficie (\%)

1. Pastizal de Hilaria mutica

2. Matorral bajo inerme de Flourensia cernua

3. Matorral mediano subinerme de Leucophyllum frutescens-Acacia rigidula

4. Matorral mediano subinerme de Acacia-Prosopis-Flourensia-Larrea

5. Matorral mediano espinoso de Prosopis-Acacia

6. Matorral alto espinoso de Prosopis-Acacia

7. Matorral alto subinerme de Helietta parvifolia

Se registraron 405 especies (incluyendo variedades y subespecies) que se distribuyen de la siguiente manera:

Familias

Angiospermas

Pteridófitas

Gimnospermas
72

2

1
Géneros

260

4

1
Especies

395

8

2

Las especies se enlistan por familias en el Cuadro 1. Se indica en qué tipo de vegetación fue encontrada cada especie y si fue observada como riparia o maleza.

Pastizal de Hilaria mutica

La fisonomía del pastizal se caracteriza por un estrato herbáceo que en la época de lluvias cubre toda la superficie del suelo y alcanza una altura hasta de $0.5 \mathrm{~m}$. El pasto amacollado $H$. mutica (zacate toboso) es la especie dominante en la comunidad y los pastos cespitosos Bouteloua trifida e Hilaria belangeri llegan a ser codominantes. Además de las gramíneas mencionadas se encuentran: Aristida pansa, A. roemeriana, Bothriochloa barbinodis var. barbinodis, Panicum hallii, Pappophorum bicolor, Setaria macrostachya, Sporobolus pulvinatus y Tridens muticus.

La carpeta herbácea se encuentra interrumpida por elementos arbustivos espaciados, no mayores de $2 \mathrm{~m}$ de altura, de los géneros Acacia, Opuntia y Prosopis, así como por Flourensia cernua y Lycium berlandieri. La composición florística del pastizal de $H$. mutica, así como la del resto de los otros tipos de vegetación, se presenta en el Apéndice. 
El pastizal de $H$. mutica se distribuye en forma de manchones en las llanuras de nula o escasa pendiente del centro y norte de la zona de estudio. Este tipo de vegetación se conoce también para Coahuila (Muller, 1947; Miranda y Hernández X., 1963b) y Chihuahua (Shreve, 1942; Rzedowski, 1978). En Coahuila, H. mutica se distribuye en suelos arcillosos húmedos con poca influencia salina en las franjas lacustres de las cuencas cerradas, a veces en manchones sobre declives con suelo arcilloso (Hernández X., 1964). Rzedowski (1978) menciona que los extensos pastizales de $H$. mutica en Chihuahua y Coahuila aparentemente no están restringidos a suelos salinos, sino que también prosperan en otros caracterizados por drenaje más o menos deficiente y textura fina.

Este pastizal es equivalente a los llanos de Hilaria (Shreve, 1942) y al pastizal halófito abierto de H. mutica (Anónimo, 1973); en parte, al zacatal edáfico (Rzedowski, 1961), a los pastizales halofíticos (Miranda y Hernández X., 1963b) y al zacatal y asociaciones de halófitos (Rojas-Mendoza, 1965).

\section{Matorral bajo inerme de Flourensia cernua}

Se trata de un matorral bajo, normalmente de menos de $1 \mathrm{~m}$ de alto, que llega a ser denso y con una cobertura mayor a $75 \%$ del área. El estrato arbustivo está dominado por Flourensia cernua (hojasén), que en ocasiones comparte la dominancia con Larrea tridentata (gobernadora). Ambas especies son micrófilas y no presentan espinas. Son frecuentes otros arbustos bajos que se agrupan en manchones de 1-2 m de altura. El estrato inferior lo dominan Hilaria mutica y/o Bouteloua trifida, alcanzando coberturas hasta de $70 \%$.

Las mayores extensiones del matorral de F. cernua se presentan en las planicies y porciones donde termina el declive de las lomas y comienza la llanura. De modo diferente se presenta sobre los lomeríos, pues se encuentra de manera interdigitada o reticulada entre las suaves depresiones, siguiendo el curso de las mismas.

Este tipo de vegetación se ha reconocido para el suroeste y noroeste de Nuevo León (Rojas-Mendoza, 1965; Anónimo, 1973) y se extiende al centro y norte de México (Muller, 1947; Gentry, 1957; Miranda y Hernández X., 1963a,b; Marroquín et al., 1964 y Rzedowski, 1961, 1978). En Coahuila, Miranda y Hernández X. (1963b), siguiendo a Muller (1947), mencionan que $F$. cernua aumenta en abundancia y aun puede llegar a formar una asociación pura en suelos profundos y bien drenados.

El matorral de $F$. cernua es equivalente en parte al central plateau desert scrub, posteriormente llamado Chihuahuan desert scrub (Muller, 1939, 1947); al matorral desértico de Chihuahua (Gentry, 1957); al matorral inerme parvifolio (Miranda y Hernández X., 1963a; Anónimo, 1973); al matorral inerme perennifolio (Miranda y Hernández X., 1963b); al matorral nanófilo subperennifolio con Larrea-Flourensia-Prosopis (Rojas-Mendoza, 1965) y al matorral desértico micrófilo (Rzedowski, 1961; Marroquín et al., 1964).

\section{Matorral mediano subinerme de Acacia-Prosopis-Flourensia-Larrea}

Esta comunidad vegetal está dominada por arbustos espinosos en el estrato superior, cuya altura oscila entre 1-2 m, y otros elementos inermes en el inferior, usualmente 
de menos de $1.5 \mathrm{~m}$ de alto. La cobertura del dosel superior es baja, $20 \%$ o menos, comparada con la del inferior que es de $60-80 \%$. Es el tipo de vegetación más heterogéneo en cuanto a sus especies dominantes. Encontramos matorrales dominados por Prosopis glandulosa en el estrato superior y por $F$. cernua en el inferior, o dominados por el primero y L. tridentata. O bien lo encontramos con Acacia rigidula (chaparro prieto) en el superior y F. cernua o L. tridentata en el inferior. También puede existir codominancia de ProsopisAcacia, así como de Flourensia-Larrea en sus respectivos estratos.

El matorral se distribuye por toda la zona en las partes planas o levemente onduladas, así como en las laderas de los lomeríos. La distribución general de este tipo de vegetación y las equivalencias nomenclaturales utilizadas por otros autores son las mismas que las ya mencionadas para el matorral de F. cernua.

Matorral mediano subinerme de Leucophyllum frutescens-Acacia rigidula

Es un matorral caracterizado por la codominancia de Leucophyllum frutescens (cenizo), arbusto inerme que proporciona una tonalidad gris a la comunidad, y por Acacia rigidula, que es un arbusto con espinas laterales. La cobertura del estrato arbustivo superior, con altura de 1-2 m, alcanza hasta 70\%, aunque suele haber áreas abiertas. El arbustivo inferior, de menos de $0.5 \mathrm{~m}$ de alto, es variable en cobertura y lo dominan tanto elementos espinosos como inermes. En este último estrato, las especies que forman manchones o montículos son las que tienen mayor cobertura, tales como Agave lechuguilla, Calliandra conferta, Chamaecrista greggii, Heliotropium torreyi, Jatropha dioica y Krameria ramosissima. Las cactáceas, poligaláceas y plantas trepadoras alcanzan su mayor diversidad en este tipo de vegetación. Se observó a Phoradendron tomentosum parasitando a Prosopis glandulosa.

El aspecto estacional en la comunidad se refleja significativamente en sus especies herbáceas. En la época seca encontramos una carpeta de Bouteloua trifida en las áreas abiertas de la vegetación, así como otras especies aparentemente asociadas a la copa de los arbustos. En período de lluvias florecen y se desarrollan una gran diversidad de plantas, tanto erguidas como postradas. En tal aspecto es posible separarlas en dos estratos: uno superior compuesto por especies de porte erecto, donde Psilostrophe gnaphalodes, Aristida roemeriana y Panicum hallii llegan a ser abundantes, y otro estrato muscinal de menos de 10 centímetros de altura, integrado por elementos de hábito postrado que forman una alfombra de diferentes colores y cuya cobertura alcanza en ocasiones a cubrir totalmente la superficie del suelo. Son notables en este estrato Gnaphalopsis micropoides, Thymophylla pentachaeta, Chaetopappa bellioides, Dalea pogonathera, Heliotropium confertifolium, Lepidium virginicum, Melampodium cinereum var. hirtellum y Tiquilia canescens.

La gran variedad de ambientes en que se desarrolla esta vegetación se manifiesta en la presencia de diferentes variantes fisonómicas. Una de ellas, que podría denominarse la "más xerófila", está caracterizada por una estratificación más marcada, cobertura más abierta y aumento en el valor de importancia de especies que aunque no exclusivas si son propias de lugares con aridez más acentuada, tales como Larrea tridentata, Flourensia cernua, Lycium berlandieri, Viguiera stenoloba, Ziziphus obtusifolia e Hilaria mutica. Otra variante, la "más mesófila", está determinada por la presencia de Yucca filifera (palma), 
que alcanza alturas hasta de $6 \mathrm{~m}$. Esta última modalidad se desarrolla sobre las faldas orientales de la Sierra de Lampazos, pues dicho macizo montañoso logra interceptar parte de la humedad que portan los vientos provenientes del mar del Golfo de México. El clima más húmedo propicia el desarrollo de un matorral más alto y exuberante en donde Cordia boissieri (anacahuita) y Karwinskia humboldtiana (coyotillo) comparten la dominancia junto con Acacia rigidula y Leucophyllum frutescens.

Este matorral mediano se localiza sobre los lomeríos y mesetas de los llanos y valles de la zona, así como lomas que bordean la Sierra de Lampazos, siguiendo los abanicos aluviales. La pendiente puede ser nula o bien suave o pronunciada y la pedregosidad y afloramientos rocosos varían desde abundantes a escasos. Se ha reconocido para el norte y oriente de Nuevo León con el nombre de matorral bajo subperennifolio con AcaciaLeucophyllum-Cordia y para el centro de Nuevo León con el nombre de matorral bajo subcaducifolio con Cordia-Acacia-Karwinskia (Rojas-Mendoza, 1965; Anónimo, 1973).

Matorral alto subinerme de Helietta parvifolia

La fisonomía de esta comunidad la proporciona el estrato arbustivo superior, cuya altura varía entre $2.5-5 \mathrm{~m}$ y alcanza una cobertura hasta de $70 \%$. Lo caracteriza Helietta parvifolia (barreta), rutácea inerme que le da a la vegetación una estructura relativamente uniforme, pues normalmente es la única dominante, aunque en ocasiones Acacia berlandieri (huajillo) es igual de importante. Las plantas prevalecientes del estrato arbustivo medio (0.5$2 \mathrm{~m}$ de alto) son Leucophyllum frutescens y Acacia rigidula. Su cobertura varía de 50 a $80 \%$. El estrato inferior, menor de $0.5 \mathrm{~m}$ de altura, es diverso tanto en especies como en cobertura; es notable en los claros de la vegetación, donde Agave lechuguilla, Euphorbia antisyphilitica (candelilla) y las gramíneas amacolladas de los géneros Bouteloua, Tridens y Aristida son los elementos más importantes. El terreno es pedregoso y es común encontrar plantas creciendo sobre rocas.

Este tipo de vegetación se encuentra en laderas, cañadas y partes altas, sean planas o con pendiente, de las mesetas y lomeríos. Crece sobre suelos someros que a veces presentan una capa superficial de hojarasca y son comunes los afloramientos de la roca madre. Es equivalente al matorral alto subinerme (González-Medrano, 1972; Anónimo, 1973) y matorral alto subperennifolio con Acacia-Pithecellobium-Helietta (Rojas-Mendoza, 1965); en parte al piedmont scrub o piedmont shrub (Muller (1939, 1947), matorral subinerme parvifolio (Miranda y Hernández X., 1963a) y matorral submontano (Rzedowski, 1961).

Matorral mediano espinoso de Prosopis-Acacia

Es un matorral que rara vez sobrepasa los $2 \mathrm{~m}$ de altura, dominado por arbustos espinosos de folíolo u hoja pequeña. La estratificación de la comunidad no es clara, aunque es posible reconocer un dosel arbustivo superior que varía entre 0.5-2 $\mathrm{m}$ de altura, en donde los dominantes son Prosopis glandulosa (mezquite) y Acacia farnesiana (huizache) o bien, el primero y Castela texana (chaparro amargoso) o Celtis pallida (granjeno). Su cobertura 
no es muy alta, pues sus elementos se encuentran espaciados. El estrato inferior, menor de $0.4 \mathrm{~m}$ de altura, lo dominan las gramíneas Hilaria mutica y Bouteloua trifida.

Se localiza en superficies planas o poco onduladas de la zona, o bien siguiendo el curso de los arroyos intermitentes. Es equivalente en parte al matorral espinoso con espinas laterales (Miranda y Hernández X., 1963a) y matorral espinoso de hojas pequeñas (Miranda y Hernández X., 1963b).

\section{Matorral alto espinoso de Prosopis-Acacia}

La fisonomía de este tipo de vegetación está determinada por el estrato superior, que varía de 2 a $8 \mathrm{~m}$ de altura. Lo domina o codomina Prosopis glandulosa (mezquite), junto con Acacia farnesiana (huizache), Acacia greggii var. wrightii (uña de gato), Acacia schaffneri var. bravoensis o Karwinskia humboldtiana; todos espinosos, excepto el último. En ocasiones es posible reconocer un estrato medio, siendo Opuntia lindheimeri (nopal) uno de los elementos más frecuentes. El inferior, de menos de $0.5 \mathrm{~m}$ de alto, fluctúa en cobertura acorde con la época del año y cobertura del estrato superior, pues lo encontramos con gran diversidad de especies (principalmente anuales) en la época lluviosa y lugares abiertos a pobremente representado en la época seca y lugares cerrados. Con gran frecuencia se encuentran plantas trepadoras como Cissus incisa, Clematis drummondii, Cocculus diversifolius e Ibervillea tenuisecta. Ocasionalmente se observó a Phoradendron tomentosum creciendo sobre Prosopis glandulosa.

Se localiza en las partes más bajas de las llanuras o valles, al borde de las represas y siguiendo los cauces de las corrientes de agua. Aparentemente se asocia con niveles freáticos altos y suelos profundos de origen aluvial. Es equivalente en parte al pastizal con Cactus-Acacia (Gentry, 1957); al mezquital extradesértico (Rzedowski, 1961); al bosque caducifolio espinoso de P. glandulosa con Opuntia lindheimeri (Anónimo, 1973); al bosque espinoso y perennifolio (Miranda y Hernández X., 1963b) y al bosque bajo micrófilo con Prosopis-Acacia-Celtis (Rojas-Mendoza, 1965).

Es difícil separar este tipo de vegetación del matorral mediano espinoso de ProsopisAcacia, sobre todo cuando este último se desarrolla sobre los arroyos intermitentes de la zona, pues además de las especies dominantes, ambos tipos de vegetación crecen bajo condiciones ecológicas similares y fisonómicamente están determinados por $P$. glandulosa, que es un elemento espinoso con follaje casi todo el año. En tal caso, la diferencia principal es la altura de la comunidad. Al respecto, Rzedowski (1978) menciona que los mezquitales de las zonas áridas se presentan en forma de matorrales xerófilos y que existen formas transicionales entre las comunidades arborescentes y arbustivas de Prosopis.

\section{AFINIDADES GEOGRÁFICAS DE LOS TIPOS DE VEGETACIÓN}

De acuerdo con su fisonomía y especies dominantes, tres comunidades de la zona estudiada son muy semejantes a la vegetación del Desierto Chihuahuense en el Altiplano Mexicano y pudieran concebirse como penetraciones hacia la misma. Además de hacerlo en Nuevo León, Hilaria mutica forma pastizales circundados por arbustos desérticos en los 
terrenos con drenaje deficiente de Coahuila y Chihuahua (Muller, 1947; Rzedowski, 1978). El matorral bajo inerme de Flourensia cernua y el matorral mediano de Acacia-ProsopisFlourensia-Larrea forman parte del Chihuahuan desert scrub (= central plateau desert scrub) descrito para Coahuila y Nuevo León (Muller 1939, 1947). El matorral de F. cernua recuerda a la variante "más pobre" del matorral desértico micrófilo de la Planicie Occidental de San Luis Potosí (Rzedowski, 1961). A su vez, las variantes aquí descritas de Prosopis y Larrea - Prosopis y Flourensia del matorral de Acacia-Prosopis-Flourensia-Larrea, aunque no presentaron un estrato de eminencias, recuerdan a la variante "más exuberante" del mismo matorral desértico micrófilo de San Luis Potosí. En la zona se encontraron las siguientes especies, que son representativas de comunidades vegetales características del Desierto Chihuahuense (Rzedowski, 1961, 1978): Condalia spathulata, Descurainia pinnata, Ephedra aspera, Hibiscus coulteri, Koeberlinia spinosa, Larrea tridentata, Lycium berlandieri, Nama parvifolium, Opuntia leptocaulis, Parthenium incanum, Tidestromia lanuginosa, Tiquilia canescens y Ziziphus obtusifolia.

En contraste con la vegetación anterior, el matorral mediano subinerme de Leucophyllum frutescens y Acacia rigidula y el matorral alto subinerme de Helietta parvifolia pueden ser vistos como extensiones de la vegetación de la Planicie Costera del Noreste. El primero forma parte del Tamaulipan thorn scrub (= eastern coastal plain scrub) descrito para Nuevo León y Coahuila (Muller, 1939, 1947) y probablemente represente una continuación del Tamaulipan thorn forest de Tamaulipas y extremo sur de Texas (Muller, 1947). El estrato arbustivo superior del matorral de L. frutescens y A. rigidula guarda semejanza, al menos en cuanto a sus componentes florísticos, con el estrato arbustivo de la variante "con menos precipitación" de la selva baja espinosa perennifolia en el nordeste de Tamaulipas (González-Medrano, 1972). La afinidad meridional y las adaptaciones xéricas de sus elementos, así como la similitud florística entre el matorral alto de $H$. parvifolia y la vegetación arbustiva de la provincia florística de la Planicie Costera del Noreste sustentan su ubicación como parte de esta última. A su vez, dichas características del matorral de H. parvifolia lo excluyen de formar parte de la provincia de la Sierra Madre Oriental, caracterizada por bosques de Quercus y Pinus de afinidad boreal (Rzedowski, 1978). El matorral alto subinerme forma parte del matorral submontano o piedmont scrub (Muller 1939, 1947; Rzedowski, 1961), común en los cerros bajos y laderas de la Sierra Madre Oriental que colindan con la Planicie Costera del Golfo en Nuevo León y Coahuila (Muller, 1939, 1947; Rojas-Mendoza, 1965). En Tamaulipas se encuentra en los lomeríos cercanos a San Fernando y Sierras aisladas de San Carlos y de Tamaulipas (González-Medrano, 1972; Briones, 1991). Siempre asociado a la Sierra Madre Oriental, sea en laderas de sotavento o barlovento, el matorral submontano se distribuye desde Coahuila (en el norte) hasta Hidalgo (en el sur), reapareciendo algo modificado en Puebla y Oaxaca (Rzedowski, 1978).

Los matorrales mediano y alto de Prosopis-Acacia constituyen una vegetación más bien ubicuista y no restringida a alguna de las dos provincias florísticas anteriores. Estos matorrales en general se distribuyen en las partes bajas de las llanuras o siguiendo los cauces de los ríos. Mejor representados y con los nombres de bosque espinoso y perennifolio o bosque de mezquite se les conoce en otras regiones semiáridas de México (Miranda y Hernández X., 1963b; Rzedowski, 1978).

Las diferencias principales entre los matorrales de la Planicie Costera del Noreste y los del Altiplano fueron ya señaladas por Muller $(1939,1947)$, al comparar la vegetación 
que describió como "Tamaulipan thorn forest" y "Chihuahuan desert shrub". Aunque ambas regiones topográficamente ocupan zonas semejantes, la menor elevación y la mayor precipitación y exposición a los vientos del Golfo de México de la Planicie Costera, se correlacionan con el desarrollo de una vegetación caracterizada por una mayor preponderancia de especies espinosas, una mayor abundancia de pastos y hierbas y un crecimiento más denso de arbustos y árboles bajos. Como diferencias florísticas importantes, Muller (1939, 1947) menciona (1) la ausencia en los matorrales de la Planicie Costera de Opuntia imbricata, Larrea divaricata, Dasylirion texanum, Agave falcata, Fouquieria splendens y (2) la presencia con gran número de individuos de Cordia boissieri, Acacia amentacea, A. wrightii, A. berlandieri, Yucca treculeana, Lippia macrostachya, Salvia ballotaeflora, Leucophyllum minus, Forestiera angustifolia, Prosopis chilense, Eysenhardtia polystachya, Opuntia spp.

Cabe mencionar que la vegetación de la zona posee afinidad florística con las planicies del sur de Texas o planicies del río Grande, caracterizadas por praderas abiertas con Prosopis glandulosa, Celtis pallida, Ziziphus obtusifolia, Aloysia gratissima, Condalia hookeri, Castela texana, Leucophyllum frutescens, Acacia farnesiana, A. rigidula, A. berlandieri y otros arbustos y árboles pequeños (Gould y Thomas, 1962; Correll y Johnston, 1970).

En conclusión, la distribución y afinidades geográficas de los tipos de vegetación encontrados caracterizan a la zona como un amplio ecotono ente las provincias florísticas de la Planicie Costera del Noreste de México y el Altiplano. La coexistencia en la zona de vegetación arbustiva característica de las tierras bajas de las planicies del Golfo junto con vegetación comúnmente encontrada en el Desierto Chihuahuense, podría explicarse en función del incremento en la aridez y el suave levantamiento de la superficie continental en sentido este-oeste y de la ausencia de accidentes topográficos notables en el sector nororiental de México.

\section{AGRADECIMIENTOS}

Se agradece el apoyo y las sugerencias del Dr. Jorge S. Marroquín de la Fuente y del M.C. Glafiro Alanís durante la fase inicial del trabajo. EL Biól. Ismael Cabral proporcionó información meteorológica de la zona. Las facilidades otorgadas por el Lic. Juan Chavéz A., del Laboratorio de Visualización de Datos del Instituto de Ecología, A.C., fueron valiosas durante la elaboración del mapa. El Dr. Joel Flores hizo aportaciones al revisar el manuscrito. Dos revisores anónimos hicieron útiles sugerencias al trabajo.

\section{LITERATURA CITADA}

Anónimo. 1973. Coeficientes de agostadero de la República Mexicana. Estado de Nuevo León. Comisión Técnico Consultiva para la Determinación Regional de los Coeficientes de Agostadero. Secretaría de Agricultura y Ganadería. México, D.F.

Anónimo. 1974, 1976, 1977. Cartas topográficas, escala 1:50 000: Progreso, Salinillas, Camarón, EI Jabalí, Lampazos de Naranjo, Ciudad Anáhuac, Valladares y San Miguel. Comisión/Dirección de Estudios del Territorio Nacional. México, D.F. 
Anónimo. 1981. Síntesis geográfica de Nuevo León y anexo cartográfico. Instituto Nacional de Estadística, Geografía e Informática. México, D.F. 170 pp. + 13 mapas.

Braun-Blanquet, J. 1979. Fitosociología. H. Blume Ediciones. 820 pp.

Briones, 0. 1991. Sobre la flora, vegetación y fitogeografía de la Sierra de San Carlos, Tamaulipas. Acta Bot. Mex. 16: 15-43.

Correll, D. S. y M. C. Johnston. 1970. Manual of the vascular plants of Texas. Texas Research Foundation. Renner, Tex. 1881 pp.

Dice, L. R. 1943. The biotic provinces of North America. Universitiy of Michigan Press. Ann Arbor. $73 \mathrm{pp}$.

Edwards, M. T. 1939. An ecological and vegetational study of Sierra Madre Oriental, México. Tesis. Graduate School. University of Texas. Austin. $144 \mathrm{pp}$.

Gentry, H. S. 1957. Los pastizales de Durango. Ediciones del Instituto Mexicano de Recursos Naturales Renovables. México, D.F. 361 pp.

González-Medrano, F. 1972. La vegetación del nordeste de Tamaulipas. An. Inst. Biol. Méx. Ser. Bot. 43: $11-50$.

Gould, F. y G. Thomas. 1962. Texas plants. A checklist and ecological summary. Texas Agric. Exp. Sta. A. \& M. College of Texas. College Station. 122 pp.

Hernández X., E. 1964. Los pastos y los pastizales. In: Las zonas áridas del centro y noreste de México y el aprovechamiento de sus recursos. Ediciones del Instituto Mexicano de Recursos Naturales Renovables. México, D.F. pp. 97-127.

Kuchler, A. W. 1967. Vegetation mapping. Ronald Press Co. Nueva York. 472 pp.

Marroquín, J., G. Borja, R. Velázquez y J. A. de la Cruz. 1964. Estudio ecológico dasonómico de las zonas áridas del norte de México. Inst. Nal. Invest. Forest. Pub. Esp. 2, Secretaría de Agricultura y Ganadería. México, D.F. 165 pp.

Miranda, F. y Hernández X., E. 1963a. Los tipos de vegetación de México y su clasificación. Bol. Soc. Bot. Méx. 28: 29-178.

Miranda, F. y Hernández X., E. 1963b. Fisiografía y vegetación. In: Las zonas áridas del centro y noreste de México y el aprovechamiento de sus recursos. Ediciones del Instituto Mexicano de Recursos Naturales Renovables. México, D.F. pp. 1-27.

Muller, C. H. 1939. Relation of the vegetation and climatic types in Nuevo Leon, Mexico. Amer. Midl. Nat. 21(3): 687-729.

Muller, C. H. 1947. Vegetation and climate in Coahuila. Madroño 9: 33-57.

Mullerried, F. 1944. Geología del estado de Nuevo León. An. Inst. Inv. Cient. UANL. Monterrey 1(1): 167-199.

Rojas-Mendoza, P. 1965. Generalidades sobre la vegetación del Estado de Nuevo León y datos acerca de su flora. Tesis doctoral. Facultad de Ciencias, Universidad Nacional Autónoma de México. México, D.F. $125+75$ pp.

Rzedowski, J. 1961. Vegetación del estado de San Luis Potosí. Tesis doctoral. Facultad de Ciencias. Universidad Nacional Autónoma de México. México, D.F. 228 pp.

Rzedowski, J. 1978. Vegetación de México. Limusa. México, D.F. 432 pp.

Shreve, F. 1942. Grassland and related vegetation in northern Mexico. Madroño 6: 190-198.

Thayer, W. H. 1916. The physiography of Mexico. Jour. Geol. 24: 61-94. 
Briones y Villarreal: Vegetación y Flora de un Ecotono entre dos Provincias Florísticas de México

\section{APÉNDICE}

Lista florística de un ecotono desértico entre las províncias florísticas del Altiplano y de la Planicie Costera del Noreste de México. El ecotono se localiza en el municipio de Lampazos de Naranjo, Nuevo León. Se registran 405 especies (incluyendo variedades y subespecies) y 75 familias de plantas vasculares. En seguida de la especie se indica el tipo de vegetación y si la especie fue registrada como riparia o maleza, de acuerdo con la siguiente clave: 1 = Pastizal de Hilaria mutica; 2 = Matorral bajo inerme de Flourensia cernua; 3 = Matorral mediano subinerme de Leucophyllum frutescens-Acacia rigidula; 4 = Matorral mediano subinerme de Acacia-Prosopis-Flourensia-Larrea; $5=$ Matorral mediano espinoso de Prosopis-Acacia; 6 = Matorral alto espinoso de Prosopis-Acacia; 7 = Matorral alto subinerme de Helietta parvifolia; $R=$ Riparia y $M=$ Maleza.

\begin{tabular}{|c|c|c|c|c|c|c|c|c|c|}
\hline & \multicolumn{9}{|c|}{ Tipo de Vegetación } \\
\hline & 1 & 2 & 3 & 4 & 5 & 6 & 7 & $\mathrm{R}$ & M \\
\hline ACANTHACEAE & & & & & & & & & \\
\hline $\begin{array}{l}\text { Anisacanthus quadrifidus (Vahl) Nees var. wrightii (Torr.) } \\
\text { Henrick. }\end{array}$ & & & $x$ & & & & & & \\
\hline Carlowrightia parviflora (Buckl.) Wasshausen & & & & & & & & & $\mathrm{x}$ \\
\hline Dyschoriste decumbens (Gray) O. Ktze. & & & & & & & $\mathrm{X}$ & & \\
\hline Ruellia nudiflora (Gray) Urban & & & & & & & & & $\mathrm{x}$ \\
\hline R. occidentalis (Gray) Tharp \& Barkley & & & $x$ & $\mathrm{X}$ & & $\mathrm{X}$ & & & \\
\hline Siphonoglossa pilosella (Nees) Torr. & & & $x$ & & & & & & \\
\hline Yeatesia platystegia (Torr.) Hilsenbeck & $x$ & & & & & & & & \\
\hline AGAVACEAE & & & & & & & & & \\
\hline Agave lechuguilla Torr. & & & $x$ & & & & & & \\
\hline A. scabra Salm-Dyck ssp. scabra & & $x$ & $x$ & & & $\mathrm{X}$ & $\mathrm{X}$ & & $x$ \\
\hline Dasylirion texanum Scheele & & & $x$ & & & & $\mathrm{x}$ & & \\
\hline Hesperaloe funifera (Koch) Trel. & & & & & & & $x$ & & \\
\hline H. parviflora (Torr.) Coult. & & & & & & & $x$ & & \\
\hline Yucca filifera Chab. & & & $x$ & & & & & & \\
\hline Y. rostrata Engelm. & & & & & & & $x$ & & \\
\hline Y. torreyi Shafer & & $x$ & $x$ & $x$ & $x$ & $x$ & $x$ & & \\
\hline AMARANTHACEAE & & & & & & & & & \\
\hline Amaranthus palmeri Wats. & & & & & & & & & $x$ \\
\hline A. berlandieri (Moq.) Uline \& Bray & & & & & & & & & $x$ \\
\hline Celosia nitida Vahl & & & & $\mathrm{X}$ & & & & & \\
\hline Froelichia arizonica Thornb. & & & $x$ & & & & & & \\
\hline Froelichia interrupta (L.) Moq. & & & & & & & & & $\mathrm{x}$ \\
\hline
\end{tabular}


Apéndice. Continuación.

\begin{tabular}{|c|c|c|c|c|c|c|c|c|c|}
\hline & \multicolumn{9}{|c|}{ Tipo de Vegetación } \\
\hline & 1 & 2 & 3 & 4 & 5 & 6 & 7 & $\mathrm{R}$ & M \\
\hline Guilleminea lanuginosa (Poir.) Hook. & & & $\mathrm{X}$ & & & & & & \\
\hline Tidestromia lanuginosa (Nutt.) Standl. var. lanuginosa & & & & & & & & & $x$ \\
\hline ANACARDIACEAE & & & & & & & & & \\
\hline Pistacia texana Swingle & & & $x$ & & & & & & \\
\hline APOCYNACEAE & & & & & & & & & \\
\hline Macrosiphonia hypoleuca (Benth.) Muell. Arg. & & & $x$ & & & & & & \\
\hline ASCLEPIADACEAE & & & & & & & & & \\
\hline Asclepias oenotheroides Cham. \& Schlecht. & & & & & & & & & $x$ \\
\hline Cynanchum barbigerum (Scheele) Shinners & & $\mathrm{x}$ & $\mathrm{X}$ & & & & & & \\
\hline Matelea sp. & & & & & & & & & $x$ \\
\hline BIGNONIACEAE & & & & & & & & & \\
\hline Chilopsis linearis (Cav.) Sweet. ssp. linearis & & & $\mathrm{X}$ & & & & $\mathrm{X}$ & & \\
\hline Tecoma stans (L.) Juss. & & & $x$ & & & & $\mathrm{x}$ & & \\
\hline BORAGINACEAE & & & & & & & & & \\
\hline Cordia boissieri A. DC. & & & $x$ & $x$ & & $x$ & $x$ & & $x$ \\
\hline Heliotropium angiospermum Murr. & & & $x$ & & & & $x$ & & $x$ \\
\hline H. confertifolium (Torr.) Gray & & & $x$ & $x$ & & & $x$ & & \\
\hline H. curassavicum L. var. curassavicum & & & & & & & & $x$ & \\
\hline H. greggii Torr. & & & & & & & & & $x$ \\
\hline H. molle (Torr.) I. M. Johnst. & $\mathrm{x}$ & & & & & & & & \\
\hline H. torreyi I. M. Johnst. & & & $x$ & & & & $x$ & & $x$ \\
\hline Omphalodes aliena Gray & & & $\mathrm{x}$ & & & $\mathrm{X}$ & & $\mathrm{x}$ & \\
\hline Tiquilia canescens (DC.) A. Richardson & & & $x$ & & & & & & \\
\hline BROMELIACEAE & & & & & & & & & \\
\hline Hechtia glomerata Zucc. & & & & & & & $x$ & & \\
\hline CACTACEAE & & & & & & & & & \\
\hline Ancistrocactus scheeri (Salm-Dyck) B. \& R. & & & $x$ & & & & $x$ & & \\
\hline Coryphantha difficilis (Quehl) Berger & & & $x$ & & & & & & \\
\hline $\begin{array}{l}\text { C. sulcata (Engelm.) B. \& R. var. nickelsiae (K. Brand.) } \\
\text { Benson }\end{array}$ & & & $x$ & & & $X$ & & & \\
\hline
\end{tabular}


Briones y Villarreal: Vegetación y Flora de un Ecotono entre dos Provincias Florísticas de México

Apéndice. Continuación.

\begin{tabular}{|c|c|c|c|c|c|c|c|c|c|}
\hline & \multicolumn{9}{|c|}{ Tipo de Vegetación } \\
\hline & 1 & 2 & 3 & 4 & 5 & 6 & 7 & $\mathrm{R}$ & M \\
\hline C. sulcata (Engelm.) B. \& R. var. sulcata & & & $x$ & & & & & & \\
\hline Echinocactus texensis Hopffer & & $x$ & $x$ & & & $\mathrm{x}$ & & & $x$ \\
\hline Echinocereus blanckii (Poselger) Palmer & & & & & & & $x$ & & \\
\hline E. conglomeratus Forst. & & & $x$ & & & & $x$ & & \\
\hline E. enneacanthus Engelm. var. enneacanthus & $x$ & $x$ & $x$ & $\mathrm{x}$ & & & $\mathrm{x}$ & & \\
\hline E. pectinatus (Scheidw.) Engelm. & & & $x$ & & & & & & \\
\hline Escobaria chaffeyi B. \& R. & & & $x$ & $x$ & & & $x$ & & \\
\hline E. chihuahuensis B. \& R. & & & $x$ & & & & & & \\
\hline E. runyonii B. \& R. & & & & & & & $x$ & & \\
\hline E. tuberculosa (Engelm.) B. \& R. & & & $x$ & & & & & & \\
\hline Ferocactus hamatacanthus (Muhl.) B. \& R. & & & & $\mathrm{x}$ & & $\mathrm{x}$ & $\mathrm{x}$ & & \\
\hline $\begin{array}{l}\text { Mammillaria gummifera Engelm. var. applanata (Engelm.) } \\
\text { Benson }\end{array}$ & & & $x$ & & & & $\mathrm{X}$ & & \\
\hline M. gummifera Engelm. var. hemisphaerica (Engelm.) Benson & & & & & & & $\mathrm{X}$ & & \\
\hline M. gummifera Engelm. var. meiacantha (Engelm.) Benson & & & $x$ & & & & & & \\
\hline Opuntia grahamii Engelm. & & & & & & & $x$ & & \\
\hline O. leptocaulis DC. & $x$ & $x$ & $x$ & $\mathrm{x}$ & $\mathrm{x}$ & $\mathrm{x}$ & $x$ & & \\
\hline O. lindheimeri Engelm. & $x$ & $x$ & $x$ & $\mathrm{x}$ & $\mathrm{x}$ & $\mathrm{x}$ & $x$ & & $\mathrm{x}$ \\
\hline Wilcoxia tuberosa (Poselger) B. \& R. & & $x$ & $x$ & & & & & & \\
\hline CAMPANULACEAE & & & & & & & & & \\
\hline Lobelia berlandieri A.DC. & & & & & & & $x$ & & \\
\hline CAPPARIDACEAE & & & & & & & & & \\
\hline Polanisia dodecandra (L.) DC. spp. riograndensis Iltis & & & & & & & & & $\mathrm{x}$ \\
\hline CARYOPHYLLACEAE & & & & & & & & & \\
\hline Stellaria media (L.) Cyr. & & & & & & $x$ & & $x$ & \\
\hline CELASTRACEAE & & & & & & & & & \\
\hline Schaefferia cuneifolia Gray & & $x$ & $x$ & $x$ & $x$ & $x$ & $x$ & & \\
\hline CHENOPODIACEAE & & & & & & & & & \\
\hline Salsola iberica Senn. \& Pau. & & & & & & & & & $x$ \\
\hline
\end{tabular}


Acta Botanica Mexicana (2001), 55: 39-67

Apéndice. Continuación.

\begin{tabular}{|c|c|c|c|c|c|c|c|c|c|}
\hline & \multicolumn{9}{|c|}{ Tipo de Vegetación } \\
\hline & 1 & 2 & 3 & 4 & 5 & 6 & 7 & $\mathrm{R}$ & M \\
\hline COCHLOSPERMACEAE & & & & & & & & & \\
\hline Amoreuxia wrigthii Gray & & & $x$ & $x$ & & & $x$ & & $\mathrm{x}$ \\
\hline COMMELINACEAE & & & & & & & & & \\
\hline Commelina erecta L. & & & $x$ & & & & & & \\
\hline COMPOSITAE & & & & & & & & & \\
\hline Acourtia runcinata (D. Don.) Turner & & & $x$ & & & & & & \\
\hline A. wrigthii (Gray) King \& Reveal & & & & & & & & & $\mathrm{x}$ \\
\hline Ambrosia artemisiifolia L. & $\mathrm{x}$ & & $\mathrm{X}$ & $\mathrm{X}$ & & $\mathrm{X}$ & & & \\
\hline A. confertiflora DC. & $x$ & & $x$ & $x$ & & $\mathrm{X}$ & & & \\
\hline Aphanostephus ramosissimus DC. var. ramosissimus & $x$ & & & & & $\mathrm{X}$ & & $x$ & $\mathrm{x}$ \\
\hline Baccharis salicifolia (Ruiz \& Pavón) Pers. & & & & & & $\mathrm{X}$ & & $x$ & \\
\hline Bahia absinthifolia Benth. & & & $x$ & $x$ & & $x$ & $x$ & & \\
\hline Calyptocarpus vialis Less. & & & $x$ & & & & & & $x$ \\
\hline Centaurea americana Nutt. & & & $x$ & & & & & & \\
\hline Chaetopappa bellioides (Gray) Shinners & & & $x$ & & & & & & $\mathrm{x}$ \\
\hline Conyza coulteri Gray & & & & & & $\mathrm{X}$ & & & \\
\hline Dichaetophora campestris Gray & & & & & & $x$ & & $x$ & \\
\hline Eclipta alba (L.) Hassk. & & & & & & & & $x$ & \\
\hline Evax verna Raf. & & & $x$ & & & & & & $\mathrm{x}$ \\
\hline Florestina tripteris DC. & & & $x$ & & & & & & $x$ \\
\hline Flourensia cernua DC. & $x$ & $\mathrm{X}$ & $x$ & $x$ & & $x$ & & & \\
\hline Gnaphalopsis micropoides DC. & & & $x$ & $x$ & & & $x$ & & $\mathrm{x}$ \\
\hline Gochnathia hypoleuca DC. & & & & & $x$ & & $x$ & & \\
\hline Grindelia oxylepis Greene & & & & & & & & & $x$ \\
\hline Gutierrezia sphaerocephala Gray & & & & & & $x$ & & & \\
\hline Gymnosperma glutinosum (Spreng.) Less. & & & $x$ & $x$ & & & & & $\mathrm{x}$ \\
\hline Helianthus annuus L. & & & & & & & & & $\mathrm{x}$ \\
\hline Helenium microcephalum DC. & & & $x$ & & & & & & \\
\hline H. quadridentatum Labill. & & & & & & $\mathrm{X}$ & & $x$ & $x$ \\
\hline Jefea brevifolia (Gray) Strother & & & $x$ & $x$ & & & $x$ & & \\
\hline
\end{tabular}


Briones y Villarreal: Vegetación y Flora de un Ecotono entre dos Provincias Florísticas de México

Apéndice. Continuación.

\begin{tabular}{|c|c|c|c|c|c|c|c|c|c|}
\hline & \multicolumn{9}{|c|}{ Tipo de Vegetación } \\
\hline & 1 & 2 & 3 & 4 & 5 & 6 & 7 & $\mathrm{R}$ & M \\
\hline Machaeranthera pinnatifida (Hook.) Shinners & & & & & & $\mathrm{x}$ & & $\mathrm{x}$ & \\
\hline Melampodium cinereum DC. var. hirtellum Stuessy & & & $x$ & & & $\mathrm{x}$ & $\mathrm{x}$ & & $x$ \\
\hline Palafoxia texana DC. var. texana & & & $x$ & & & & & $x$ & $\mathrm{x}$ \\
\hline Parthenium confertum Gray & & & $x$ & $x$ & & $\mathrm{x}$ & & & $x$ \\
\hline P. incanum H.B.K. & & & $x$ & & & & & & \\
\hline Pectis angustifolia Torr. var. tenella (DC.) Keil & & & $x$ & & & & & & \\
\hline Pinaropappus roseus (Less.) Less. & & & & & & & $\mathrm{X}$ & & \\
\hline Pluchea purpurascens (Sw.) DC. & & & & & & $\mathrm{X}$ & & $x$ & \\
\hline Psilostrophe gnaphalodes DC. & $x$ & $x$ & $x$ & $\mathrm{x}$ & & $\mathrm{x}$ & & & $x$ \\
\hline Ratibida columnifera (Nutt.) Woot. \& Standl. & & & $x$ & & & & & & \\
\hline Sanvitalia ocymoides DC. & & & $x$ & $\mathrm{x}$ & & & & & $x$ \\
\hline Senecio flaccidus Less. var. flaccidus & & & & $\mathrm{X}$ & & & & & $\mathrm{x}$ \\
\hline S. glabellus Poir. & & & $x$ & & & & & & \\
\hline Simsia calva (Engelm. \& Gray) Gray & & & $x$ & & & & $\mathrm{x}$ & & \\
\hline Sonchus oleraceus L. & & & & & & $\mathrm{x}$ & & & $\mathrm{X}$ \\
\hline Thymophylla pentachaeta (DC.) Small & $\mathrm{x}$ & & $\mathrm{x}$ & $\mathrm{x}$ & & $\mathrm{x}$ & & & \\
\hline T. pentachaeta (DC.) Small var. hartwegii (Gray) Strother & & & & & & & & & $x$ \\
\hline T. pentachaeta (DC.) Small var. pentachaeta & & & & & & & & & $x$ \\
\hline T. pentachaeta (DC.) Small var. puberula (Rydb.) Strother & & & & & & & $x$ & & \\
\hline T. setifolia Lag. & & & $x$ & & & & & & \\
\hline T. tenuiloba (DC.) Small var. tenuiloba & & & & & & & & & $x$ \\
\hline T. tenuiloba (DC.) Small var. treculii (Gray) Strother & & & & & & $\mathrm{X}$ & & $x$ & \\
\hline Trixis californica Kell. & & & & & & & $x$ & & \\
\hline Verbesina encelioides (Cav.) Gray & & & & & & & & $x$ & \\
\hline V. microptera DC. & & & $x$ & & & & & & $\mathrm{X}$ \\
\hline Viguiera stenoloba Blake & $\mathrm{X}$ & $x$ & $x$ & $\mathrm{x}$ & $\mathrm{x}$ & $\mathrm{x}$ & & & \\
\hline Xanthium strumarium L. & & & & $\mathrm{X}$ & & & & & \\
\hline Wedelia acapulcensis Kunth var. hispida (Kunth) Strother & & & & & & & & & $\mathrm{X}$ \\
\hline CONVOLVULACEAE & & & & & & & & & \\
\hline Convolvulus equitans Benth. & & & & & & & & & $x$ \\
\hline
\end{tabular}


Acta Botanica Mexicana (2001), 55: 39-67

Apéndice. Continuación.

\begin{tabular}{|c|c|c|c|c|c|c|c|c|c|}
\hline & \multicolumn{9}{|c|}{ Tipo de Vegetación } \\
\hline & 1 & 2 & 3 & 4 & 5 & 6 & 7 & $\mathrm{R}$ & M \\
\hline Cuscuta cuspidata Benth. & & & & & & & & & $x$ \\
\hline Dichondra micrantha Urban & & & & & & $x$ & & $x$ & \\
\hline Evolvulus alsinoides L. var. hirticaulis Torr. & & & $x$ & $x$ & & & $x$ & & $x$ \\
\hline Ipomoea costellata Torr. & & & $x$ & & & & & & $x$ \\
\hline I. nil (L.) Roth & & & & & & & & & $x$ \\
\hline 1. aff. rupicola House & & & & & & & $x$ & & \\
\hline \multicolumn{10}{|l|}{ CRUCIFERAE } \\
\hline Descurainia pinnata (Walt.) Britt. & & & & & & $\mathrm{x}$ & & $\mathrm{X}$ & $x$ \\
\hline Draba cuneifolia Nutt. & & & $x$ & & & & & & \\
\hline Lepidium virginicum $\mathrm{L}$. & & & & & & & & & $x$ \\
\hline Lesquerella argyraea (Gray) Wats. ssp. argyraea & & & & & & & & & $x$ \\
\hline Nerisyrenia camporum (Gray) Greene & & & $x$ & & & $x$ & & $x$ & \\
\hline Sibara runcinata (Wats.) Roll. & & & $x$ & & & & & & \\
\hline Sisymbrium irio L. & & & & & & & & & $x$ \\
\hline \multicolumn{10}{|l|}{ CUCURBITACEAE } \\
\hline Cucurbita foetidissima H.B.K. & & & & & & & & & $x$ \\
\hline Ibervillea tenuisecta (Gray) Small & $x$ & & $x$ & $x$ & & $x$ & $x$ & & $x$ \\
\hline \multicolumn{10}{|l|}{ CYPERACEAE } \\
\hline Cyperus alternifolius L. & $x$ & & & & & & & & \\
\hline Cyperus sp. & & & & & & & & & $x$ \\
\hline Eleocharis albida Torr. & & & & & & & & & $x$ \\
\hline E. caribaea (Rottb.) Blake & & & & & & & & $x$ & \\
\hline E. parishii Britt. & & & & & & & & $x$ & \\
\hline Fuirena simplex Vahl & & & & & & & & $x$ & \\
\hline \multicolumn{10}{|l|}{ EBENACEAE } \\
\hline Diospyros texana Scheele & & & $x$ & & & & $x$ & & \\
\hline \multicolumn{10}{|l|}{ EPHEDRACEAE } \\
\hline Ephedra aspera Engelm. & & $x$ & $x$ & & & & & & \\
\hline E. pedunculata Engelm. & & & $x$ & & & & & & $x$ \\
\hline
\end{tabular}


Briones y Villarreal: Vegetación y Flora de un Ecotono entre dos Provincias Florísticas de México Apéndice. Continuación.

\begin{tabular}{|c|c|c|c|c|c|c|c|c|c|}
\hline & \multicolumn{9}{|c|}{ Tipo de Vegetación } \\
\hline & 1 & 2 & 3 & 4 & 5 & 6 & 7 & $\mathrm{R}$ & M \\
\hline \multicolumn{10}{|l|}{ EUPHORBIACEAE } \\
\hline Acalypha monostachya Cav. & & & & & & & $x$ & & \\
\hline A. rhomboidea Raf. & & & $x$ & & & & & & \\
\hline Argythamnia neomexicana Muell. & & & $x$ & & & & & & $x$ \\
\hline Bernardia myricaefolia (Scheele) Wats. & & & $x$ & & & & $x$ & & \\
\hline Croton dioicus Cav. & & & $x$ & & & & & & \\
\hline C. lindheimerianus Scheele & & & & & & & & & $\mathrm{x}$ \\
\hline C. incanus H.B.K. & & & $x$ & & & & $\mathrm{x}$ & & $x$ \\
\hline Euphorbia albomarginata T. \& G. & & & & & & $x$ & & & \\
\hline E. antisyphilitica Zucc. & & & $x$ & $x$ & & & $x$ & & \\
\hline E. cinerascens Engelm. & & & $x$ & & & & & & \\
\hline E. cordifolia Ell. & & & $x$ & & & & & $\mathrm{x}$ & \\
\hline E. cyathophora Murr. & & & & & & & $\mathrm{X}$ & $x$ & \\
\hline E. dentata Michx. & & & & & & & & & $x$ \\
\hline E. golondrina Wheeler & & & $x$ & & & & $x$ & & \\
\hline E. maculata L. & & & & & & & & & $x$ \\
\hline E. micromera Engelm. & & & $x$ & & & & & & \\
\hline E. prostrata Ait. & & & $x$ & & & & & $x$ & \\
\hline E. revoluta Engelm. & & & & & & & & & $x$ \\
\hline E. aff. serrula Engelm. & & & & $x$ & & & & & $x$ \\
\hline Jatropha cathartica Terán \& Berl. & $x$ & & & & & & & & \\
\hline J. dioica Cerv. & $\mathrm{x}$ & $x$ & $x$ & $x$ & $\mathrm{X}$ & $\mathrm{x}$ & $x$ & & \\
\hline Phyllanthus polygonoides Spreng. & & & $x$ & & & & $x$ & & $x$ \\
\hline Ricinus communis $\mathrm{L}$. & & & & & & $\mathrm{X}$ & & & \\
\hline Stillingia spinulosa Torr. & & & & $x$ & & & & & \\
\hline \multicolumn{10}{|l|}{ FAGACEAE } \\
\hline Quercus laceyi Small & & & & & & & $x$ & & \\
\hline \multicolumn{10}{|l|}{ GENTIANACEAE } \\
\hline Centaurium calycosusm (Buckl.) Fer. & & & $x$ & & & & & & \\
\hline
\end{tabular}


Apéndice. Continuación.

\begin{tabular}{|c|c|c|c|c|c|c|c|c|c|}
\hline & \multicolumn{9}{|c|}{ Tipo de Vegetación } \\
\hline & 1 & 2 & 3 & 4 & 5 & 6 & 7 & $\mathrm{R}$ & M \\
\hline GRAMINEAE & & & & & & & & & \\
\hline Agrostis semiverticillata (Forsk.) Christ. & & & & & & & & $\mathrm{x}$ & \\
\hline Aristida adscensionis L. & & & $x$ & $\mathrm{x}$ & & & $x$ & & $x$ \\
\hline A. longiseta Steud. & & & & & & & & & $x$ \\
\hline A. longiseta Steud. var. rariflora Hitchc. & & & $x$ & & & & $x$ & & \\
\hline A. pansa Woot. \& Standl. & $x$ & $x$ & & & $x$ & & & & \\
\hline A. roemeriana Scheele & $x$ & & $x$ & & & & $x$ & & $x$ \\
\hline Arundo donax L. & & & & & & & & $\mathrm{x}$ & \\
\hline Bothriochloa barbinodis (Lag.) Herter var. barbinodis & $x$ & & & & & & & & \\
\hline Bouteloua curtipendula (Michx.) Torr. & & & $x$ & & & & $x$ & & \\
\hline B. radicosa (Fourn.) Griffiths & & & $x$ & & & & $x$ & & \\
\hline B. trifida Thurb. & $x$ & $x$ & $x$ & $x$ & $x$ & $x$ & $x$ & & $x$ \\
\hline Buchloe dactyloides (Nutt.) Engelm. & & & & & & & & & $x$ \\
\hline Cenchrus ciliaris L. & & & $x$ & $x$ & & $x$ & & & $x$ \\
\hline C. echinatus $\mathrm{L}$. & & & $x$ & $x$ & & & & & \\
\hline C. incertus M. A. Curtis & & & & & & & & & $x$ \\
\hline Chloris cucullata Bisch. & & & & & & $x$ & & & \\
\hline C. pluriflora (Fourn.) Clayton & & & & & & $x$ & & & \\
\hline Cynodon dactylon (L.) Pers. & & & & & & $\mathrm{X}$ & & $\mathrm{x}$ & $x$ \\
\hline Digitaria californica (Benth.) Henr. & & $x$ & $x$ & $x$ & & $x$ & & & \\
\hline D. cognata (Schult.) Pilger & & & $x$ & & & & & & \\
\hline D. hitchcockii (Chase) Stuck. & & & & & & & $x$ & & \\
\hline Dichanthium sericeum A. Camus & & & & & & $\mathrm{x}$ & & & \\
\hline Echinochloa colona (L.) Link & & & & & & & & & $x$ \\
\hline Eragrostis barrelieri Daveau & & & & & & & & & $x$ \\
\hline E. cilianensis (All.) E. Mosher & & & $x$ & & & & & & \\
\hline E. curtipedicellata Buckl. & & & & & & & & & $x$ \\
\hline E. intermedia Hitch. & & & & & & & & & $x$ \\
\hline Erioneuron pilosum (Buckl.) Nash & & & $x$ & & & & & & \\
\hline E. pulchellum (H.B.K.) Tateoka & & & $x$ & $x$ & & & & & \\
\hline
\end{tabular}


Briones y Villarreal: Vegetación y Flora de un Ecotono entre dos Provincias Florísticas de México

Apéndice. Continuación.

\begin{tabular}{|c|c|c|c|c|c|c|c|c|c|}
\hline & \multicolumn{9}{|c|}{ Tipo de Vegetación } \\
\hline & 1 & 2 & 3 & 4 & 5 & 6 & 7 & $\mathrm{R}$ & M \\
\hline Heteropogon contortus (L.) Beauv. & & & $x$ & & $x$ & & & & \\
\hline Hilaria belangeri (Steud.) Nash & $x$ & & & & & & & & \\
\hline H. mutica (Buckl.) Benth. & $x$ & $x$ & $x$ & $x$ & $x$ & & & & \\
\hline Panicum fasciculatum Sw. & & & & & & & & & $x$ \\
\hline P. flexile (Gatt.) Scribn. & & & & $\mathrm{X}$ & & & & & \\
\hline P. hallii Vasey & $x$ & & $x$ & $\mathrm{x}$ & & & $\mathrm{x}$ & & $x$ \\
\hline Pappophorum bicolor Fourn. & $x$ & $\mathrm{X}$ & $x$ & & & $\mathrm{X}$ & $x$ & & \\
\hline Rhynchelytrum repens (Willd.) C. E. Hubb. & & & & & & & & & $x$ \\
\hline Setaria grisebachii Fourn. & & & & & & & $x$ & & \\
\hline S. leucopila (Scribn \& Merr.) K. Schum. ? & & & & & & & $\mathrm{x}$ & & \\
\hline S. macrostachya H.B.K. & $x$ & & $x$ & $x$ & $x$ & $\mathrm{x}$ & & & $\mathrm{x}$ \\
\hline S. ramiseta (Scribn.) Pilger & & & & & & $\mathrm{X}$ & & & \\
\hline S. scheelei (Steud.) Hitchc. & & & & & & & & & $\mathrm{x}$ \\
\hline S. texana Emery & & & & $\mathrm{x}$ & & $x$ & & $x$ & $x$ \\
\hline Sorghum halepense (L.) Pers. & & & & & & & & $\mathrm{x}$ & \\
\hline Sporobolus pulvinatus Swallen & $x$ & & & & & & & & \\
\hline Tragus berteronianus Schult. & & & $x$ & & & & & & \\
\hline Tridens albescens (Vasey) Woot \& Standl. & & & $x$ & & & $\mathrm{X}$ & & & $\mathrm{X}$ \\
\hline T. eragrostoides (Vasey \& Scribn.) Nash & & & $x$ & $\mathrm{x}$ & & $\mathrm{X}$ & & & \\
\hline T. flavus (L.) Hitchc. var. chapmanii (Small) Shinners & & $\mathrm{x}$ & $x$ & & & & $x$ & & $x$ \\
\hline T. muticus (Torr.) Nash var. muticus & $x$ & $x$ & $x$ & $\mathrm{x}$ & & $\mathrm{x}$ & $x$ & & $x$ \\
\hline T. texanus (S. Wats.) Nash & & & $x$ & & & & & & $x$ \\
\hline Triticum aestivum L. & & & & & & & & & $x$ \\
\hline HYDROPHYLLACEAE & & & & & & & & & \\
\hline Nama parvifolium (Torr.) Greenm. & & & $x$ & $\mathrm{x}$ & & & & & $x$ \\
\hline N. undulatum H.B.K. & & & & & & & & & $x$ \\
\hline Phacelia congesta Hook. & & & $x$ & & & & & & \\
\hline P. patuliflora (Engelm. \& Gray) Gray & & & $x$ & & & & & & \\
\hline KOEBERLINIACEAE & & & & & & & & & \\
\hline Koeberlinia spinosa Zucc. & $x$ & $x$ & $x$ & $\mathrm{X}$ & $\mathrm{x}$ & $\mathrm{x}$ & & & \\
\hline
\end{tabular}


Apéndice. Continuación.

\begin{tabular}{|c|c|c|c|c|c|c|c|c|c|}
\hline & \multicolumn{9}{|c|}{ Tipo de Vegetación } \\
\hline & 1 & 2 & 3 & 4 & 5 & 6 & 7 & $\mathrm{R}$ & M \\
\hline KRAMERIACEAE & & & & & & & & & \\
\hline Krameria ramosissima (Gray) Wats. & & $\mathrm{X}$ & $x$ & $\mathrm{X}$ & & & $x$ & & \\
\hline LABIATAE & & & & & & & & & \\
\hline Hedeoma drummondii Benth. & & & $x$ & & & & $x$ & & \\
\hline Salvia ballotaeflora Benth. & & & $x$ & $X$ & & $\mathrm{x}$ & & & $x$ \\
\hline S. texana (Scheele) Torr. & & & $\mathrm{x}$ & & & & & & \\
\hline LEGUMINOSAE & & & & & & & & & \\
\hline Acacia berlandieri Benth. & & & $\mathrm{x}$ & $\mathrm{X}$ & & & $x$ & & \\
\hline A. farnesiana (L.) Willd. var. farnesiana & $x$ & & $x$ & & $x$ & $x$ & $x$ & & $x$ \\
\hline A. greggii Gray var. wrigthii (Benth.) Isely & $x$ & $x$ & $x$ & & $x$ & $x$ & & & \\
\hline A. rigidula Benth. & $x$ & $x$ & $x$ & $\mathrm{x}$ & $\mathrm{x}$ & $\mathrm{x}$ & $x$ & & $x$ \\
\hline A. roemeriana Scheele & & & & & & & $x$ & & \\
\hline A. schaffneri (Wats.) Herm. var. bravoensis Isely & & & & & & $\mathrm{x}$ & & & \\
\hline Astragalus nuttallianus DC. & & & & & & & & & $x$ \\
\hline A. wootonii Sheld. & & & & $\mathrm{x}$ & & & & & \\
\hline Bauhinia uniflora Wats. & & & $\mathrm{x}$ & & & & & & \\
\hline Calliandra conferta Benth. & & & $\mathrm{x}$ & & & $\mathrm{x}$ & & & \\
\hline Cercidium macrum I. M. Johnst. & $\mathrm{x}$ & & $\mathrm{x}$ & $\mathrm{X}$ & & $x$ & & & \\
\hline C. texanum Gray & $\mathrm{x}$ & $\mathrm{x}$ & $\mathrm{X}$ & $\mathrm{x}$ & $\mathrm{X}$ & $x$ & & & $\mathrm{x}$ \\
\hline Chamaecrista greggii (Gray) Heller & & & $\mathrm{x}$ & & & & $x$ & & $\mathrm{x}$ \\
\hline Cracca purpurea L. & & & $x$ & & & & & & \\
\hline Dalea hospes (Rose) Bullock & & & & & & & & & $x$ \\
\hline D. lanata Spreng. & & & $x$ & & & & & & \\
\hline D. nana Torr. & & & & & & & & & $x$ \\
\hline D. pogonathera Gray & & $x$ & $x$ & $\mathrm{x}$ & & & & & $x$ \\
\hline D. wrightii Gray & & & $x$ & & & & & & \\
\hline $\begin{array}{l}\text { Desmanthus virgatus (L.) Willd. var. depressus (Willd.) } \\
\text { B. L. Turner }\end{array}$ & & & & $x$ & & $x$ & & & $x$ \\
\hline Eysenhardtia texana Scheele & $\mathrm{X}$ & $\mathrm{x}$ & $\mathrm{X}$ & $x$ & $\mathrm{x}$ & $\mathrm{X}$ & $\mathrm{x}$ & & $x$ \\
\hline Hoffmannseggia glauca (Ort.) Eifert & $x$ & & & & & & & & \\
\hline
\end{tabular}


Briones y Villarreal: Vegetación y Flora de un Ecotono entre dos Provincias Florísticas de México

Apéndice. Continuación.

\begin{tabular}{|c|c|c|c|c|c|c|c|c|c|}
\hline & \multicolumn{9}{|c|}{ Tipo de Vegetación } \\
\hline & 1 & 2 & 3 & 4 & 5 & 6 & 7 & $\mathrm{R}$ & M \\
\hline Lupinus aff. texensis Hook. & & & & & & & & & $x$ \\
\hline Nissolia platycalyx Wats. & & & $x$ & & & & & & $x$ \\
\hline Parkinsonia aculeata L. & & & & & & & & & $x$ \\
\hline Macroptilium atropurpureum (DC.) Urban & & & & & & & $x$ & & \\
\hline Prosopis glandulosa Torr. & $\mathrm{X}$ & $x$ & $x$ & $\mathrm{X}$ & $x$ & $\mathrm{X}$ & & & $x$ \\
\hline P. reptans Benth. var. cinerascens (Gray) Burk. & $x$ & $x$ & & $\mathrm{x}$ & & & & & $x$ \\
\hline Schrankia latidens (Small) Schum. & & & & & & & $\mathrm{x}$ & & \\
\hline Senna bauhinoides (Gray) Irwin \& Barneby & & & $x$ & & & $\mathrm{X}$ & & & $x$ \\
\hline S. lindheimeriana (Scheele) Irwin \& Barneby & & & $x$ & & & & & & \\
\hline Sophora secundiflora (Ort.) Lag. & & & $x$ & & & & & & \\
\hline LINACEAE & & & & & & & & & \\
\hline Linum rupestre (Gray) Gray & & & $x$ & & & & & & $x$ \\
\hline L. vernale (Woot.) Small & & & $x$ & & & & & & \\
\hline LOASACEAE & & & & & & & & & \\
\hline Cevallia sinuata Lag. & & & $x$ & $x$ & & $\mathrm{x}$ & & & \\
\hline Eucnide bartonioides Zucc. & & & & & & & $x$ & & \\
\hline Mentzelia sp. & & & & & & & & & $x$ \\
\hline LYTHRACEAE & & & & & & & & & \\
\hline Heimia salicifolia Link & & & & & & $\mathrm{X}$ & & $x$ & \\
\hline MALPIGHIACEAE & & & & & & & & & \\
\hline Aspicarpa hyssopifolia Gray & & & $x$ & & & & & & \\
\hline Janusia gracilis Gray & & & $x$ & & & & & & \\
\hline Mascagnia lilacina ( Wats.) Niedenzu & & & & & & & $\mathrm{x}$ & & \\
\hline MALVACEAE & & & & & & & & & \\
\hline Abutilon grandiflorum Hochr. & & & $x$ & & & & & & \\
\hline A. hypoleucum Gray & & & & & & & $x$ & & \\
\hline A. malacum Wats. & & & $x$ & $x$ & & & & & $x$ \\
\hline A. sonorae Gray & & & $x$ & & & & & & $x$ \\
\hline A. wrightii Gray & & & $x$ & & & & & & $x$ \\
\hline A. sp. & & & & & $\mathrm{x}$ & $x$ & & & \\
\hline
\end{tabular}


Acta Botanica Mexicana (2001), 55: 39-67

Apéndice. Continuación.

\begin{tabular}{|c|c|c|c|c|c|c|c|c|c|}
\hline & \multicolumn{9}{|c|}{ Tipo de Vegetación } \\
\hline & 1 & 2 & 3 & 4 & 5 & 6 & 7 & $\mathrm{R}$ & M \\
\hline Anoda wrigthii Gray & $x$ & & & & & & & & \\
\hline Herissantia crispa (L.) Brizicky & & & $x$ & & & & & & \\
\hline Hibiscus coulteri Gray & & & $x$ & & & & & & \\
\hline H. martianus Zucc. & & & & $x$ & & & $x$ & & \\
\hline Malva parviflora L. & & & $x$ & & & & & & $x$ \\
\hline Malvastrum coromandelianum (L.) Gke. & & & $x$ & & & $\mathrm{X}$ & & & $\mathrm{x}$ \\
\hline Meximalva filipes (Gray) Fryxell & & & $x$ & $\mathrm{x}$ & & & $\mathrm{x}$ & & \\
\hline Sida abutifolia Mill. & $x$ & & $x$ & $x$ & & $\mathrm{X}$ & & & $x$ \\
\hline S. tragiifolia Gray & & & & & & & & & $x$ \\
\hline Sphaeralcea hastulata Gray & & & & & & & & & $\mathrm{X}$ \\
\hline MARTYNIACEAE & & & & & & & & & \\
\hline Proboscidea louisianica (Mill.) Thell. & & & & & & & & & $\mathrm{x}$ \\
\hline MENISPERMACEAE & & & & & & & & & \\
\hline Cocculus diversifolius DC. & & & & & & $x$ & & & $x$ \\
\hline NYCTAGINACEAE & & & & & & & & & \\
\hline Acleisanthes longiflora Gray & & & $x$ & $x$ & & & & & $x$ \\
\hline A. obtusa (Choisy) Standl. & $x$ & & $x$ & & & & & & \\
\hline Allionia incarnata L. & $\mathrm{x}$ & & $x$ & $x$ & & & & & $\mathrm{x}$ \\
\hline Boerhaavia coccinea Mill. & & & $x$ & & & & & & $\mathrm{X}$ \\
\hline B. erecta L. & & & & & & & & & $\mathrm{x}$ \\
\hline Cyphomeris gypsophiloides (Mart. \& Gal.) Standl. & & & $x$ & & & & & & $\mathrm{x}$ \\
\hline Nyctaginia capitata Choisy & & & & & & & & & $\mathrm{x}$ \\
\hline Mirabilis sp. & & & $x$ & & & & & & \\
\hline NYMPHAEACEAE & & & & & & & & & \\
\hline Nymphaea odorata Ait. & & & & & & & & $\mathrm{x}$ & \\
\hline OLEACEAE & & & & & & & & & \\
\hline Forestiera angustifolia Torr. & $x$ & & $x$ & $\mathrm{x}$ & & & $x$ & & \\
\hline Fraxinus berlandieriana A. DC. & & & & & & & & $\mathrm{x}$ & \\
\hline Menodora heterophylla Moric. & & $\mathrm{x}$ & $x$ & $x$ & & $\mathrm{x}$ & & $x$ & $\mathrm{x}$ \\
\hline M. longiflora Gray & $x$ & & & & & & $x$ & & \\
\hline
\end{tabular}


Briones y Villarreal: Vegetación y Flora de un Ecotono entre dos Provincias Florísticas de México Apéndice. Continuación.

\begin{tabular}{|c|c|c|c|c|c|c|c|c|c|}
\hline & \multicolumn{9}{|c|}{ Tipo de Vegetación } \\
\hline & 1 & 2 & 3 & 4 & 5 & 6 & 7 & $\mathrm{R}$ & M \\
\hline M. scabra Gray & & & & $\mathrm{x}$ & & & & & \\
\hline M. scabra var. ramosissima Steyerm. & & & $x$ & & & & & & \\
\hline ONAGRACEAE & & & & & & & & & \\
\hline Calylophus hartwegii (Benth.) Raven & & & $x$ & & & & & & $x$ \\
\hline Gaura boquillensis Raven \& Gregory & & & & & & & & & $\mathrm{X}$ \\
\hline Oenothera kunthiana (Spach) Munz & & & $x$ & & & & & & \\
\hline OXALIDACEAE & & & & & & & & & \\
\hline Oxalis dichondraefolia Gray & & & $x$ & & & & & & $x$ \\
\hline Oxalis sp. & $x$ & & & & & & & & \\
\hline PAPAVERACEAE & & & & & & & & & \\
\hline Argemone sanguinea Greene & & & & & & & & $x$ & $x$ \\
\hline PASSIFLORACEAE & & & & & & & & & \\
\hline Passiflora tenuiloba Engelm. & & & $x$ & & & & & & $x$ \\
\hline PHYTOLACCACEAE & & & & & & & & & \\
\hline Phaulothamnus spinescens Gray & & & & & & $\mathrm{X}$ & & & \\
\hline Rivina humilis $\mathrm{L}$. & $\mathrm{x}$ & & $x$ & & & & & $x$ & $x$ \\
\hline PLANTAGINACEAE & & & & & & & & & \\
\hline Plantago virginica $\mathrm{L}$. & & & $x$ & & & & & $x$ & $x$ \\
\hline POLEMONIACEAE & & & & & & & & & \\
\hline Gilia incisa Benth. & & & $x$ & $x$ & & & & $x$ & $x$ \\
\hline POLYGALACEAE & & & & & & & & & \\
\hline Polygala glandulosa H.B.K. & & & $x$ & & & & & & \\
\hline P. hemipterocarpa Gray & & & $x$ & & & & & & \\
\hline P. lindheimeri Gray & & & $x$ & & & & & & \\
\hline P. ovatifolia Gray & & & $x$ & & & & & & $x$ \\
\hline POLYGONACEAE & & & & & & & & & \\
\hline Eriogonum sp. & & & & & & & & & $x$ \\
\hline Rumex maritimus L. & & & & & & & & $x$ & \\
\hline POLYPODIACEAE & & & & & & & & & \\
\hline Adiantum capillus-veneris $\mathrm{L}$. & & & & & & & & $x$ & \\
\hline
\end{tabular}


Apéndice. Continuación.

\begin{tabular}{|c|c|c|c|c|c|c|c|c|c|}
\hline & \multicolumn{9}{|c|}{ Tipo de Vegetación } \\
\hline & 1 & 2 & 3 & 4 & 5 & 6 & 7 & $\mathrm{R}$ & M \\
\hline Cheilanthes horridula Maxon & & & $x$ & & & & $x$ & & \\
\hline $\begin{array}{l}\text { Notholaena candida (Mart. \& Gal.) Hook. var. copelandii } \\
\text { (Hall) Tryon }\end{array}$ & & & & & & & $\mathrm{x}$ & & \\
\hline N. schaffneri (Fourn.) Underw. var. nealleyi (Coult) Weath. & & & $x$ & & & & $x$ & & \\
\hline N. sinuata (Sw.) Kaulf. var. integerrima Hook. & & & $x$ & & & & $x$ & & \\
\hline N. sinuata (Sw.) Kaulf. var. sinuata & & & & & & & $x$ & & \\
\hline PORTULACACEAE & & & & & & & & & \\
\hline Portulaca pilosa L. & $\mathrm{X}$ & $\mathrm{X}$ & $\mathrm{x}$ & $x$ & & $x$ & & & $\mathrm{X}$ \\
\hline Talinum aff. aurantiacum Engelm. & & & & & & & $x$ & & \\
\hline PRIMULACEAE & & & & & & & & & \\
\hline Anagallis arvensis $\mathrm{L}$. & & & & & & $x$ & & $x$ & $x$ \\
\hline Samolus cuneatus Small & & & & & & $x$ & & $x$ & \\
\hline S. parviflorus Raf. & & & & & & $x$ & & $x$ & \\
\hline RANUNCULACEAE & & & & & & & & & \\
\hline Clematis drummondii $\mathrm{T}$. \& G. & & & & & & $x$ & & & \\
\hline RHAMNACEAE & & & & & & & & & \\
\hline Colubrina greggii Wats. & & & $x$ & & & & $x$ & & \\
\hline C. texensis (T. \& G.) Gray & & & & & & $x$ & & & \\
\hline Condalia hookeri M.C. Johnst. var. hookeri & & & $x$ & & & $x$ & & & \\
\hline C. spathulata Gray & $x$ & $\mathrm{x}$ & $x$ & $x$ & $x$ & $x$ & & & \\
\hline Karwinskia humboldtiana Zucc. & $x$ & & $x$ & $x$ & & $x$ & $x$ & & $x$ \\
\hline Ziziphus obtusifolia (T. \& G.) Gray & $x$ & $\mathrm{X}$ & $\mathrm{X}$ & $x$ & $x$ & $x$ & & & \\
\hline ROSACEAE & & & & & & & & & \\
\hline $\begin{array}{l}\text { Vauquelinia corymbosa H. \& B. ssp. heterodon (I. M. Johnst.) } \\
\text { Hess. \& Henrick. }\end{array}$ & & & & & & & $\mathrm{X}$ & & \\
\hline RUBIACEAE & & & & & & & & & \\
\hline Galium aparine L. & & & & & & & & $x$ & \\
\hline G. proliferum Gray & & & $x$ & & & & & & \\
\hline Hedyotis nigricans (Lam.) Fosb. & $x$ & & & & & & & & $x$ \\
\hline RUTACEAE & & & & & & & & & \\
\hline Amyris madrensis Wats. & & & & & & & $x$ & & \\
\hline
\end{tabular}


Briones y Villarreal: Vegetación y Flora de un Ecotono entre dos Provincias Florísticas de México

Apéndice. Continuación.

\begin{tabular}{|c|c|c|c|c|c|c|c|c|c|}
\hline & \multicolumn{9}{|c|}{ Tipo de Vegetación } \\
\hline & 1 & 2 & 3 & 4 & 5 & 6 & 7 & $\mathrm{R}$ & M \\
\hline Helietta parvifolia (Hemsl.) Benth. & & & $x$ & & & & $X$ & & \\
\hline Thamnosma texana (Gray) Torr. & & & $x$ & $\mathrm{X}$ & & & & & \\
\hline Zanthoxylum fagara (L.) Sarg. & & & $x$ & $\mathrm{x}$ & & & & & \\
\hline SAPINDACEAE & & & & & & & & & \\
\hline Neopringlea integrifolia (Hemsl.) Wats. & & & $x$ & & & & $\mathrm{x}$ & & \\
\hline Serjania incisa Torr. & & & & & & & $\mathrm{X}$ & & \\
\hline SAPOTACEAE & & & & & & & & & \\
\hline Bumelia celastrina H.B.K. & & & & & & & $\mathrm{x}$ & & \\
\hline SCROPHULARIACEAE & & & & & & & & & \\
\hline Bacopa monnieri (L.) Wettst. & & & & & & & & $x$ & \\
\hline Leucophyllum frutescens (Berl.) I. M. Johnst. & $x$ & $x$ & $x$ & $\mathrm{x}$ & $x$ & $\mathrm{X}$ & $x$ & & $x$ \\
\hline Veronica peregrina L. var. xalapensis (H.B.K.) Penn. & & & $x$ & & & & & & \\
\hline SELAGINELLACEAE & & & & & & & & & \\
\hline Selaginella lepidophylla (Hook. \& Grev.) Spring & & & & & & & & $\mathrm{x}$ & \\
\hline S. pilifera A.Br. & & & & & & & $x$ & & \\
\hline SIMAROUBACEAE & & & & & & & & & \\
\hline Castela erecta Turp. ssp. texana (T. \& G.) Cronq. & $x$ & $x$ & $x$ & $\mathrm{X}$ & $x$ & $\mathrm{X}$ & & & \\
\hline SOLANACEAE & & & & & & & & & \\
\hline Calibrachoa parviflora (Juss.) D’Arcy & & & & & & & & $\mathrm{x}$ & \\
\hline Chamaesaracha coniodes (Dun.) Britt. & & & $x$ & & & $\mathrm{X}$ & & & $x$ \\
\hline C. crenata Rydb. & & & & & & & & & $x$ \\
\hline Lycium berlandieri Dun. & $x$ & $x$ & $x$ & $\mathrm{X}$ & & $\mathrm{X}$ & & & \\
\hline Nicotiana glauca Grah. & & & & & & $\mathrm{X}$ & & $\mathrm{X}$ & \\
\hline N. longiflora Cav. & & & $x$ & & & & & & \\
\hline N. repanda Willd. & & & & & & & & $\mathrm{X}$ & \\
\hline Physalis hederaefolia Gray & & & $x$ & & & & & & \\
\hline P. cinerascens (Dun.) A. Hitchc. & & & & & & & & & $x$ \\
\hline Quincula lobata (Torr.) Raf. & & & & & & & & $\mathrm{X}$ & $x$ \\
\hline Solanum tenuipes Bartlett var. tenuipes & & & & $\mathrm{X}$ & & & & & \\
\hline S. elaeagnifolium Cav. & $x$ & & & $\mathrm{X}$ & & $\mathrm{X}$ & & & \\
\hline
\end{tabular}


Acta Botanica Mexicana (2001), 55: 39-67

Apéndice. Continuación.

\begin{tabular}{|c|c|c|c|c|c|c|c|c|c|}
\hline & \multicolumn{9}{|c|}{ Tipo de Vegetación } \\
\hline & 1 & 2 & 3 & 4 & 5 & 6 & 7 & $\mathrm{R}$ & M \\
\hline S. rostratum Dun. & & & & & & & & & $\mathrm{X}$ \\
\hline S. triquetrum Cav. & & & & & & & & & $\mathrm{X}$ \\
\hline STERCULIACEAE & & & & & & & & & \\
\hline Melochia pyramidata L. & & & & & & & & & $\mathrm{x}$ \\
\hline Waltheria indica L. & & & & & & & & & $\mathrm{X}$ \\
\hline TURNERACEAE & & & & & & & & & \\
\hline Turnera diffusa Willd. var. aphrodisiaca (Ward.) Urban & & & $\mathrm{X}$ & & & & & & \\
\hline ULMACEAE & & & & & & & & & \\
\hline Celtis laevigata Willd. & $\mathrm{X}$ & & $\mathrm{x}$ & $\mathrm{X}$ & $\mathrm{X}$ & $x$ & & & \\
\hline C. reticulata Torr. & & & & & & $\mathrm{X}$ & & & \\
\hline UMBELLIFERAE & & & & & & & & & \\
\hline Ammoselinum popei T. \& G. & & & $x$ & & & & & & $\mathrm{x}$ \\
\hline Eryngium nasturtiifolium Juss. & & & & & & & & $x$ & \\
\hline Hydrocotyle verticillata Thunb. & & & & & & & & $x$ & \\
\hline Torilis arvensis (Huds.) Link & & & $x$ & & & & & & \\
\hline URTICACEAE & & & & & & & & & \\
\hline Parietaria pennsylvanica Muhl. & & & $x$ & & & $x$ & & $x$ & \\
\hline Urtica urens L. & & & & & & & & $x$ & \\
\hline VERBENACEAE & & & & & & & & & \\
\hline Aloysia gratissima (Gill. \& Hook.) Troncoso & $x$ & $\mathrm{x}$ & & $x$ & $x$ & & & & \\
\hline A. gratissima var. schulzae (Standl.) Moldenke & & & $x$ & & & $x$ & $x$ & & \\
\hline A. macrostachya (Torr.) Moldenke & & & $\mathrm{X}$ & & & & & & \\
\hline Citharexylon brachyanthum (Gray) Gray & & $x$ & $x$ & $x$ & & $x$ & $\mathrm{x}$ & & \\
\hline Lantana camara L. & & & $\mathrm{x}$ & & & $\mathrm{X}$ & & & $\mathrm{x}$ \\
\hline L. horrida H.B.K. & & & $\mathrm{X}$ & & & $\mathrm{X}$ & & & \\
\hline L. macropoda Torr. & & $\mathrm{X}$ & $x$ & $x$ & & & & & $\mathrm{x}$ \\
\hline Lippia graveolens H.B.K. & & $\mathrm{X}$ & $x$ & & & $x$ & $x$ & & $\mathrm{x}$ \\
\hline Phyla nodiflora (L.) Greene & & & & & & & & $x$ & \\
\hline P. lanceolata (Michx.) Greene & & & & & & & & $\mathrm{X}$ & \\
\hline Tetraclea coulteri Gray & $x$ & & & & & & & & \\
\hline
\end{tabular}


Briones y Villarreal: Vegetación y Flora de un Ecotono entre dos Provincias Florísticas de México Apéndice. Continuación.

\begin{tabular}{|l|l|l|l|l|l|l|l|l|l|}
\hline & \multicolumn{5}{|c|}{ Tipo de Vegetación } \\
\hline Verbena bipinnatifida Nutt. & 1 & 2 & 3 & 4 & 5 & 6 & 7 & $\mathrm{R}$ & $\mathrm{M}$ \\
\hline V. canescens H.B.K. & & & & & & & & $\mathrm{X}$ & \\
\hline V. halei Small & & & $\mathrm{X}$ & $\mathrm{X}$ & & $\mathrm{X}$ & & & $\mathrm{X}$ \\
\hline V. quadrangulata Heller & & & & & & $\mathrm{X}$ & & $\mathrm{X}$ & \\
\hline VISCACEAE & & & $\mathrm{X}$ & & & & & $\mathrm{X}$ & \\
\hline Phoradendron tomentosum (DC.) Gray & & & & & & & & \\
\hline VITACEAE & & & $\mathrm{X}$ & & & $\mathrm{X}$ & & & \\
\hline Cissus incisa (Nutt.) Des Moul. & & & & & & & & & \\
\hline ZYGOPHYLLACEAE & & & $\mathrm{X}$ & & & $\mathrm{X}$ & & & $\mathrm{X}$ \\
\hline Kallstroemia californica (Wats.) Vail & & & $\mathrm{X}$ & $\mathrm{X}$ & & & & & $\mathrm{X}$ \\
\hline Larrea tridentata (DC.) Cav. & $\mathrm{X}$ & $\mathrm{X}$ & $\mathrm{X}$ & $\mathrm{X}$ & & & $\mathrm{X}$ & & $\mathrm{X}$ \\
\hline Peganum mexicanum Gray & & & & & & & & & $\mathrm{X}$ \\
\hline Guaiacum angustifolium Engelm. & $\mathrm{X}$ & $\mathrm{X}$ & $\mathrm{X}$ & $\mathrm{X}$ & $\mathrm{X}$ & $\mathrm{X}$ & $\mathrm{X}$ & $\mathrm{X}$ & $\mathrm{X}$ \\
\hline
\end{tabular}




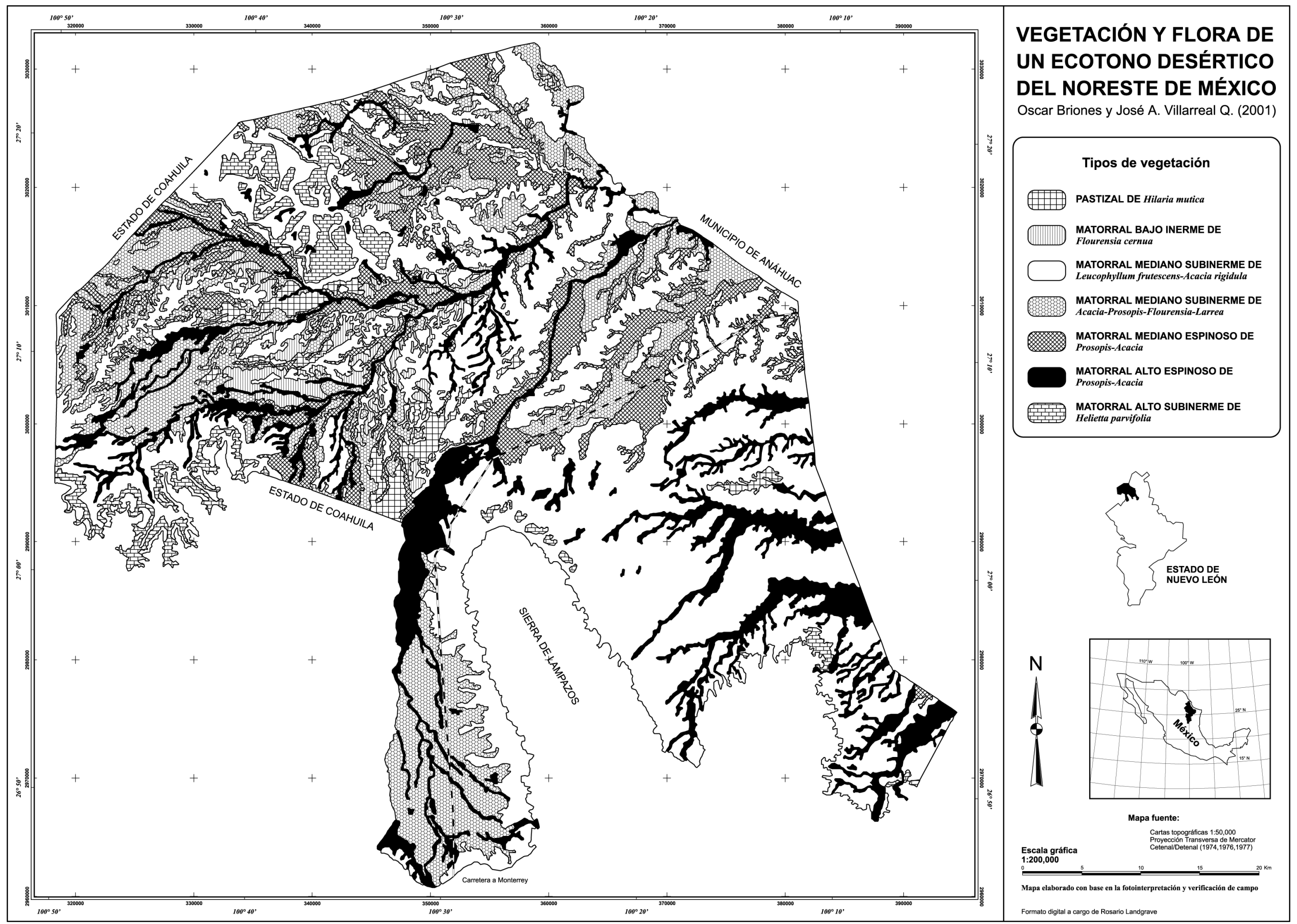

